TRANSACTIONS OF THE

AMERICAN MATHEMATICAL SOCIETY

Volume 357, Number 6, Pages 2445-2465

S 0002-9947(04)03598-6

Article electronically published on October 28, 2004

\title{
WEIGHTED REARRANGEMENT INEQUALITIES FOR LOCAL SHARP MAXIMAL FUNCTIONS
}

\author{
ANDREI K. LERNER
}

\begin{abstract}
Several weighted rearrangement inequalities for uncentered and centered local sharp functions are proved. These results are applied to obtain new weighted weak-type and strong-type estimates for singular integrals. A self-improving property of sharp function inequalities is established.
\end{abstract}

\section{INTRODUCTION}

This paper continues the study of the rearrangement inequalities in terms of sharp maximal functions $\mathrm{BK}, \mathrm{BDS}, \mathrm{L1}, \mathrm{L2}$. Let $f_{\omega}^{*}(t)$ denote the non-increasing rearrangement of $f$ with respect to a weight $\omega$, and let $f_{\omega}^{* *}(t)=t^{-1} \int_{0}^{t} f_{\omega}^{*}(\tau) d \tau$. Throughout the paper, a weight is supposed to be a non-negative locally integrable function. Given a measurable set $E$, let $\omega(E)=\int_{E} \omega(x) d x$. Given a cube $Q \subset \mathbb{R}^{n}$, consider the (weighted) Fefferman-Stein [FS2] and so-called local [JT, Str] sharp maximal functions relative to $Q$ defined by

$$
f_{\omega ; Q}^{\#}(x)=\sup _{x \in Q^{\prime} \subset Q} \inf _{c \in \mathbb{R}} \frac{1}{\omega\left(Q^{\prime}\right)} \int_{Q^{\prime}}|f(y)-c| \omega(y) d y
$$

and

$$
M_{\lambda, \omega ; Q}^{\#} f(x)=\sup _{x \in Q^{\prime} \subset Q} \inf _{c \in \mathbb{R}}\left((f-c) \chi_{Q^{\prime}}\right)_{\omega}^{*}\left(\lambda \omega\left(Q^{\prime}\right)\right) \quad(0<\lambda \leq 1),
$$

respectively, where the supremum is taken over all cubes $Q^{\prime} \subset Q$ containing $x$. When $Q \equiv \mathbb{R}^{n}$ or $\omega$ is Lebesgue measure we drop the subscripts $Q$ or $\omega$, respectively.

Both definitions (1.1) and (1.2) are closely related to the space $B M O$ [JN]. Indeed, the sharp function $f^{\#}$ is directly generated by the definition of $B M O$ :

$$
\|f\|_{B M O}=\left\|f^{\#}\right\|_{\infty},
$$

while the local sharp function $M_{\lambda}^{\#} f$ is generated by an alternate characterization of $B M O$ :

$$
\lambda\left\|M_{\lambda}^{\#} f\right\|_{\infty} \leq\|f\|_{B M O} \leq c_{n}\left\|M_{\lambda}^{\#} f\right\|_{\infty} \quad(0<\lambda \leq 1 / 2) .
$$

The first estimate in (1.3) trivially holds by Chebyshev's inequality, while the second one is a deep result due to John [Jo] and Strömberg [Str].

Besides their relation to $B M O$, sharp functions are also a very useful tool in studying many important operators arising in harmonic analysis. They can be used

Received by the editors September 10, 2003 and, in revised form, December 4, 2003.

2000 Mathematics Subject Classification. Primary 42B20, 42B25.

Key words and phrases. Weighted rearrangements, sharp maximal functions, singular integrals.

(C)2004 American Mathematical Society 
for a pointwise control of singular integrals, commutators, multipliers, LittlewoodPaley and pseudo-differential operators (see, e.g., [ABKP, [AP], CFF2], CP2] [Ja], $\mathrm{JT}, \mathrm{Ku}$, L1]). We will be concerned only with applications of sharp function inequalities to classical singular integrals $T=p . v . f * K$ with kernel $K \in C^{1}$ outside of the origin satisfying

$$
\|\widehat{K}\|_{\infty} \leq c, \quad|\nabla K(x)| \leq c /|x|^{n+1} .
$$

For any appropriate $f$ and all $x \in \mathbb{R}^{n}$ (see [JT], L1]),

$$
M_{\lambda}^{\#}(T f)(x) \leq c_{\lambda, n} M f(x) \quad(0<\lambda<1),
$$

where $M f$ is the Hardy-Littlewood maximal function.

Rearrangement estimates relating an arbitrary function $f$ and its sharp function reflect a twofold role of sharp functions: on one hand, such estimates are useful in some questions concerning $B M O$, and, on the other hand, they serve for connection between different operators. We first recall a well-known result by Bennett, DeVore, and Sharpley ([BDS] or [BS, p. 377]) saying that for any integrable function $f$ on a cube $Q \subset \mathbb{R}^{n}$,

$$
\left(f \chi_{Q}\right)^{* *}(t) \leq c_{n}\left(f_{Q}^{\#}\right)^{*}(t)+\left(f \chi_{Q}\right)^{*}(t) \quad(0<t<|Q| / 6) .
$$

This inequality easily implies several fundamental results, for instance, the JohnNirenberg inequality [JN] or the Fefferman-Stein theorem ([FS2] or [St p. 148]) about the equivalence of the $L^{p}$-norms of $f$ and $f^{\#}, 1<p<\infty$. Also it has applications in the interpolation theory. A weighted variant of (1.5), for $A_{\infty}$-weights, was obtained in $\mathrm{BK}$.

In L1 L2, it was shown that a more precise inequality than (1.5) holds, namely, the difference of rearrangements is estimated by the local sharp maximal function $M_{\lambda}^{\#} f$ : for any measurable function $f$, any weight $\omega$, and each cube $Q \subset \mathbb{R}^{n}$,

$$
\left(f \chi_{Q}\right)_{\omega}^{*}(t) \leq 2\left(M_{\lambda_{n}, \omega ; Q}^{\#} f\right)_{\omega}^{*}(2 t)+\left(f \chi_{Q}\right)_{\omega}^{*}(2 t) \quad\left(0<t \leq \lambda_{n} \omega(Q)\right),
$$

where $\lambda_{n}$ is some constant depending only on $n$. This result was first proved for doubling weights [L1], and recently it has been observed [L2] that the doubling condition can be removed. By a standard argument, (1.6) yields the following strong-type estimate:

$$
\int_{\mathbb{R}^{n}}|f|^{p} \omega d x \leq c_{p} \int_{\mathbb{R}^{n}}\left(M_{\lambda_{n}, \omega}^{\#} f\right)^{p} \omega d x \quad(0<p<\infty),
$$

whenever $\omega\left(\mathbb{R}^{n}\right)=\infty$ and $f_{\omega}^{*}(\infty)=0$. Note, however, that such an "absolutely weighted" estimate is not quite satisfactory for applications. Inequality (1.4), for example, suggests that to get weighted inequalities for singular integrals, it is very desirable to have the unweighted local sharp function $M_{\lambda}^{\#} f$ in place of its weighted variant $M_{\lambda, \omega}^{\#} f$ on the right-hand sides of (1.6) and (1.7). A simple argument shows that $M_{\lambda, \omega}^{\#} f \asymp M_{\lambda^{\prime}}^{\#} f$ for any $A_{\infty}$-weight $\omega$ (see [L1]). In this case putting $T f$ in place of $f$ in (1.7) and applying (1.4), we get a classical inequality due to Coifman and Fefferman [CF1]. In the case of arbitrary weights the situation is much more complicated (see, e.g., [Pe], [W4] for estimates of singular integrals).

In [L3], the author used (1.6) in the unweighted case and an atomic-like decomposition of $\omega$ to prove the following estimate for any measurable $f$ with $f^{*}(\infty)=0$ 
and any weight $\omega$ :

$$
\int_{\mathbb{R}^{n}}|f(x)| \omega(x) d x \leq c_{n} \int_{\mathbb{R}^{n}} M_{\lambda_{n}}^{\#} f(x) M \omega(x) d x .
$$

By a recent extrapolation theorem of Cruz-Uribe and Pérez [CP1], (1.8) is extended to the range $1<p<\infty$ in several different ways:

$$
\int_{\mathbb{R}^{n}}|f|^{p} \omega d x \leq c_{p, n} \int_{\mathbb{R}^{n}}\left(M_{\lambda_{n}}^{\#} f\right)^{p}(M \omega / \omega)^{p} \omega d x \quad(1 \leq p<\infty)
$$

and

$$
\int_{\mathbb{R}^{n}}|f|^{p} \omega d x \leq c_{p, n} \int_{\mathbb{R}^{n}}\left(M_{\lambda_{n}}^{\#} f\right)^{p} M^{[p]+1} \omega d x \quad(1<p<\infty),
$$

where $M^{k}=M \cdot M \ldots M$ is the $k$ th iterate of $M$, and $[p]$ denotes the largest integer less than or equal to $p$. Inequality (1.9) combined with (1.4) gives a new weighted strong-type estimate for singular integrals:

$$
\int_{\mathbb{R}^{n}}|T f|^{p} \omega d x \leq c_{p, n} \int_{\mathbb{R}^{n}}(M f)^{p}(M \omega / \omega)^{p} \omega d x \quad(1 \leq p<\infty) .
$$

A natural question arises as to whether one can obtain a weighted rearrangement estimate implying (1.8). Observe that the difference $f_{\omega}^{*}(t)-f_{\omega}^{*}(2 t)$ cannot be estimated by $\left(M_{\lambda_{n}}^{\#} f\right)_{M \omega}^{*}(t)$ (the rearrangement of $M_{\lambda_{n}}^{\#} f$ with respect to $M \omega$ ), since this yields (1.10) with $M \omega$ in place of $M^{[p]+1} \omega$ for any $p \geq 1$. But it is known that $(1.10)$ is sharp in the sense that $M^{[p]+1} \omega$ cannot be replaced even by $M^{[p]} \omega$ for $p>1$ (see [L3]). By a similar reason, $f_{\omega}^{*}(t)-f_{\omega}^{*}(2 t)$ cannot be estimated by $\left(M_{\lambda_{n}}^{\#} f M \omega / \omega\right)_{\omega}^{*}(t)$, since this gives (1.9) for all $p>0$. But (1.9) for $0<p<1$ is incorrect; it suffices to take $\omega=\chi_{(0,1)}$ and $f_{N}$ such that $\left|f_{N}\right| \geq N$ on $(0,1)$ and $\left\|f_{N}\right\|_{B M O} \leq c$ for any $N$.

Our first result, proved in Section 3, says that the desired estimate, somewhat surprisingly, is a generalization of the Bennett-DeVore-Sharpley inequality (1.5). More precisely, we first prove a local variant of (1.8) (see Theorem 3.1] below), and then combine it with a covering argument of [MMNO] and an argument used in proving (1.5) to get the following.

Theorem 1.1. For any measurable $f$, any weight $\omega$, and each cube $Q \subset \mathbb{R}^{n}$,

$$
\left(f \chi_{Q}\right)_{\omega}^{* *}(t) \leq c_{n}\left(\left(M_{\lambda_{n} ; Q}^{\#} f \frac{M_{Q} \omega}{\omega}\right) \chi_{Q}\right)_{\omega}^{* *}(t)+\left(f \chi_{Q}\right)_{\omega}^{*}(t) \quad(0<t<\omega(Q) / 2)
$$

As a simple corollary, we obtain a new weighted weak-type estimate for singular integrals:

$$
(T f)_{\omega}^{* *}(t) \leq c_{n} \int_{t}^{\infty}(M f M \omega / \omega)_{\omega}^{* *}(s) \frac{d s}{s} \quad(t>0) .
$$

Note also that (1.11) yields a direct proof of (1.9) without extrapolation. Besides, (1.11) contains (1.5) as a particular case when $\omega$ is Lebesgue measure (see Section 3).

While Theorem 1.1 provides a rearrangement estimate implying (1.8) and (1.9), inequality (1.10) still depends on the extrapolation argument. This can be explained by a more delicate structure of the weight $M^{[p]+1} \omega$ in comparison with $(M \omega / \omega)^{p} \omega$. We believe that (1.10) cannot be directly obtained by means of rearrangements.

We would like to point out that a covering argument of [MMNO] allows us to get a full analogue of (1.5)):

$$
\left(f \chi_{Q}\right)_{\omega}^{* *}(t) \leq c_{n}\left(f_{\omega ; Q}^{\#}\right)_{\omega}^{*}(t)+\left(f \chi_{Q}\right)_{\omega}^{*}(t) \quad(0<t<\omega(Q) / 2)
$$


(we will not prove this fact, since the proof is essentialy the same as in [BDS]). Further, Theorem 3.1 below, in particular, gives the following pointwise estimate:

$$
f_{\omega ; Q}^{\#}(x) \leq c_{n} M_{\omega ; Q}\left(M_{\lambda_{n} ; Q}^{\#} M_{Q} \omega / \omega\right)(x) .
$$

This estimate combined with (1.12) can be used to deduce Theorem 1.1 without any restriction on $\omega$ only in the one-dimensional case. It follows from the fact that $\left(M_{\omega ; Q} f\right)_{\omega}^{*}(t) \asymp\left(f \chi_{Q}\right)_{\omega}^{* *}(t)$ if and only if the operator $M_{\omega}$ is of weak type $(1,1)$ (see [AKMP), which is true without any restriction on $\omega$ only when $n=1$.

In Section 4, we prove that inequalities (1.9) and (1.10) have a self-improving property in the sense that they imply the same inequalities only with $M f$ in place of $f$ on the left-hand side for $1<p<\infty$ :

$$
\int_{\mathbb{R}^{n}}(M f)^{p} \omega d x \leq c_{p, n} \int_{\mathbb{R}^{n}}\left(M_{\lambda_{n}}^{\#} f\right)^{p}(M \omega / \omega)^{p} \omega d x
$$

and

$$
\int_{\mathbb{R}^{n}}(M f)^{p} \omega d x \leq c_{p, n} \int_{\mathbb{R}^{n}}\left(M_{\lambda_{n}}^{\#} f\right)^{p} M^{[p]+1} \omega d x .
$$

In the unweighted case this is quite standard in view of the boundedness of $M$ in $L^{p}$ for $p>1$. However, in the weighted case the situation is different [FS1]. Our argument relies on the Fefferman-Stein inequalities [FS1] and on a pointwise relation between the Hardy-Littlewood and the local sharp maximal functions.

It is well known that in the non-doubling setting centered maximal functions (that is, those maximal functions in which the corresponding supremum is taken over cubes centered at $x$ ) have much better mapping properties than their uncentered variants. For instance, the weighted centered Hardy-Littlewood maximal function $\widetilde{M}_{\omega} f$ is of weak type $(1,1)$ for all $n \geq 1$, and hence, $\left(\widetilde{M}_{\omega} f\right)_{\omega}^{*}(t) \leq c_{n} f_{\omega}^{* *}(t)$ (cf. AKMP). However, the converse inequality $f_{\omega}^{* *}(t) \leq c\left(\widetilde{M}_{\omega} f\right)_{\omega}^{*}(t)$ in general is not true even in the case $n=1$. Indeed, take, for example, $f=\chi_{(0,1)}$ and $\omega(x)=e^{|x|}$. Then $\widetilde{M}_{\omega} f(x) \asymp e^{-2|x|}$, and thus $\widetilde{M}_{\omega} f$ belongs to $L_{\omega}^{1}$, which implies integrability of $\left(\widetilde{M}_{\omega} f\right)_{\omega}^{*}(t)$. But this contradicts the fact that $f_{\omega}^{* *}(t)$ is not integrable on $(0, \infty)$. By the same reason, the sharp function $f_{\omega ; Q}^{\#}$ cannot be replaced by its centered variant on the right-hand side of (1.12). Nevertheless, we show that inequality (1.6) in the case $Q \equiv \mathbb{R}^{n}$ can be improved by replacing $M_{\lambda_{n}, \omega}^{\#} f$ by its centered variant $\widetilde{M}_{\lambda_{n}, \omega}^{\#} f$. The main result of Section 5 is the following.

Theorem 1.2. For any measurable function $f$ and any weight $\omega$,

$$
f_{\omega}^{*}(t) \leq 2\left(\widetilde{M}_{\lambda_{n}, \omega}^{\#} f\right)_{\omega}^{*}(t / 2)+f_{\omega}^{*}(2 t) \quad\left(0<t<\lambda_{n} \omega\left(\mathbb{R}^{n}\right)\right) .
$$

As a corollary, we get (1.7) with $\widetilde{M}_{\lambda_{n}, \omega}^{\#} f$ in place of $M_{\lambda_{n}, \omega}^{\#} f$, which also improves [MMNO, Theorem 8] where the strong-type inequality with the centered sharp function $\widetilde{f}_{\omega}^{\#}$ was obtained. Further, we establish an estimate of $\widetilde{M}_{\lambda_{n}, \omega}^{\#} f$ by the unweighted local sharp function and the maximal function $P_{\lambda} \omega$ measuring " $A_{\infty}$-ness" introduced by Wilson [W1] (see also [W2]-W4]). After that we apply Theorem 1.2 to get some new weighted weak-type and strong-type inequalities for singular integrals.

Some words about the notation. For two quantities $a, b$, we write $a \asymp b$ if there exist absolute constants $c_{1}, c_{2}$ such that $c_{1} a \leq b \leq c_{2} a$. Next, $Q$ will always denote an open cube with sides parallel to the coordinate axes. Its diameter is denoted 
by $\operatorname{diam}(Q)$. Given a cube $Q$ and $r>0, r Q$ will denote the cube with the same center as $Q$ and such that $\operatorname{diam}(r Q)=r \operatorname{diam}(Q)$. For a measurable set $E \subset \mathbb{R}^{n}$, by $|E|$ we denote its Lebesgue measure. As usual, $L_{\omega}^{p}$ denotes the space of all $f$ for which $\|f\|_{L_{\omega}^{p}} \equiv\left(\int_{\mathbb{R}^{n}}|f|^{p} \omega d x\right)^{1 / p}<\infty$. The letters $c_{n}, \lambda_{n}, c_{p, n}$, etc. will denote constants depending only on $n, p$ and $n$, etc., which might change from occurrence to occurrence.

\section{Preliminaries}

2.1. Rearrangements. Given a measurable function $f$ on $\mathbb{R}^{n}$, define its nonincreasing rearrangement $f_{\omega}^{*}$ with respect to a weight $\omega$ by (cf. [CR, p. 32])

$$
f_{\omega}^{*}(t)=\sup _{\omega(E)=t} \inf _{x \in E}|f(x)| \quad\left(0<t<\omega\left(\mathbb{R}^{n}\right)\right) .
$$

Observe that the rearrangement defined in such a way is left-continuous. We will mainly use several well-known properties of rearrangements [BS, p. 41, 53]:

$$
\begin{gathered}
(f+g)_{\omega}^{*}\left(t_{1}+t_{2}\right) \leq f_{\omega}^{*}\left(t_{1}\right)+g_{\omega}^{*}\left(t_{2}\right) \quad\left(t_{1}, t_{2} \geq 0\right), \\
\left|f_{k}\right| \uparrow|f| \omega \text {-a.e. } \Rightarrow\left(f_{k}\right)_{\omega}^{*} \uparrow f_{\omega}^{*}(t) \text { and }\left(f_{k}\right)_{\omega}^{* *}(t) \uparrow f_{\omega}^{* *}(t),
\end{gathered}
$$

and

$$
\sup _{\omega(E)=t} \int_{E}|f(x)| \omega(x) d x=\int_{0}^{t} f_{\omega}^{*}(\tau) d \tau \quad(t>0) .
$$

Also conditions like $f_{\omega}^{*}(\infty)=0$ will appear often. The following simple proposition clarifies the sense of such conditions.

Proposition 2.1. Let $\omega$ be any weight such that $\omega\left(\mathbb{R}^{n}\right)=\infty$. Then $f_{\omega}^{*}(\infty)=0$ iff the distribution function $\mu_{f, \omega}(\alpha)=\omega\{x:|f(x)|>\alpha\}$ is finite for any $\alpha>0$.

Proof. Suppose that $\mu_{f, \omega}\left(\alpha_{0}\right)=\infty$ for some $\alpha_{0}>0$. Then it follows easily from the definition of the rearrangement that $f_{\omega}^{*}(t) \geq \alpha_{0}$ for all $t>0$. Therefore the condition $f_{\omega}^{*}(\infty)=0$ implies $\mu_{f, \omega}(\alpha)<\infty$ for all $\alpha>0$.

Conversely, assume $f_{\omega}^{*}(t) \geq \xi>0$ for any $t>0$. This means that $\mu_{f, \omega}(\xi)=\infty$. Thus, the condition $\mu_{f, \omega}(\alpha)<\infty, \alpha>0$, implies $f_{\omega}^{*}(\infty)=0$.

2.2. Local maximal functions and median values. It is well known that one of the constants $c$ minimizing the functional $\int_{Q}|f-c| d x$ (which appears in the definition of $\left.f^{\#}\right)$ is the mean value of $f$ over $Q$, namely $f_{Q}=|Q|^{-1} \int_{Q} f$. For the functional $\left((f-c) \chi_{Q}\right)^{*}(\lambda|Q|), 0<\lambda \leq 1 / 2$, the same role is played by a median value of $f$ over $Q$, namely by a, possibly nonunique, real number $m_{f}(Q)$ such that

$$
\left|\left\{x \in Q: f(x)>m_{f}(Q)\right\}\right| \leq|Q| / 2 \quad \text { and } \quad\left|\left\{x \in Q: f(x)<m_{f}(Q)\right\}\right| \leq|Q| / 2 .
$$

Indeed, it follows easily from its definition that

$$
\left|m_{f}(Q)\right| \leq\left(f \chi_{Q}\right)^{*}(|Q| / 2) ;
$$

moreover, in the case when $f$ is a non-negative function we can take

$$
m_{f}(Q)=\left(f \chi_{Q}\right)^{*}(|Q| / 2) .
$$

Next, it is clear that $m_{f}(Q)-c=m_{f-c}(Q)$ for any constant $c$, and hence

$$
\left|m_{f}(Q)-c\right| \leq\left((f-c) \chi_{Q}\right)^{*}(|Q| / 2)
$$


which in turn gives

$$
\left(\left(f-m_{f}(Q)\right) \chi_{Q}\right)^{*}(\lambda|Q|) \leq 2 \inf _{c}\left((f-c) \chi_{Q}\right)^{*}(\lambda|Q|) \quad(0<\lambda \leq 1 / 2) .
$$

Exactly in the same way one can define a weighted median value. Note also that in the case $\omega\left(\mathbb{R}^{n}\right)<\infty$ a weighted median value of $f$ over $\mathbb{R}^{n}$ can be defined as a number $m_{f, \omega}$ such that

$\omega\left\{x \in \mathbb{R}^{n}: f(x)>m_{f, \omega}\right\} \leq \omega\left(\mathbb{R}^{n}\right) / 2$ and $\omega\left\{x \in \mathbb{R}^{n}: f(x)<m_{f, \omega}\right\} \leq \omega\left(\mathbb{R}^{n}\right) / 2$.

Define the centered weighted local sharp function $\widetilde{M}_{\lambda, \omega}^{\#} f$ by

$$
\widetilde{M}_{\lambda, \omega}^{\#} f(x)=\sup _{Q \ni x} \inf _{c}\left((f-c) \chi_{Q}\right)_{\omega}^{*}(\lambda \omega(Q)) \quad(0<\lambda<1),
$$

where the supremum is taken over all cubes centered at $x$.

Proposition 2.2. For any weight $\omega$ with $\omega\left(\mathbb{R}^{n}\right)<\infty$ and any measurable $f$,

$$
\left(f-m_{f, \omega}\right)_{\omega}^{*}\left(\lambda \omega\left(\mathbb{R}^{n}\right)\right) \leq 4 \inf _{x \in \mathbb{R}^{n}} \widetilde{M}_{\lambda, \omega}^{\#} f(x) \quad(0<\lambda \leq 1 / 4) .
$$

Proof. Given a point $x \in \mathbb{R}^{n}$, let $Q(x, r)$ be the cube centered at $x$ of diameter $r$. By (2.2) and by the left-continuity of $f_{\omega}^{*}$,

$$
\left(f-m_{f, \omega}\right)_{\omega}^{*}\left(\lambda \omega\left(\mathbb{R}^{n}\right)\right)=\lim _{r \rightarrow \infty}\left(\left(f-m_{f, \omega}\right) \chi_{Q(x, r)}\right)_{\omega}^{*}(\lambda \omega(Q(x, r))) .
$$

Next, applying (2.1) gives

$$
\begin{aligned}
\left|m_{f, \omega}-m_{f, \omega}(Q(x, r))\right| & \leq\left(f-m_{f, \omega}(Q(x, r))\right)_{\omega}^{*}\left(\omega\left(\mathbb{R}^{n}\right) / 2\right) \\
& \leq\left(\left(f-m_{f, \omega}(Q(x, r))\right) \chi_{Q(x, r)}\right)_{\omega}^{*}\left(\omega\left(\mathbb{R}^{n}\right) / 4\right) \\
& +\left(\left(f-m_{f, \omega}(Q(x, r))\right) \chi_{\mathbb{R}^{n} \backslash Q(x, r)}\right)_{\omega}^{*}\left(\omega\left(\mathbb{R}^{n}\right) / 4\right) .
\end{aligned}
$$

For $r$ big enough, $\omega\left(\mathbb{R}^{n} \backslash Q(x, r)\right)<\omega\left(\mathbb{R}^{n}\right) / 4$, and hence the term in (2.5) will be equal to zero. Therefore, using (2.4), we obtain

$$
\begin{aligned}
\left(f-m_{f, \omega}\right)_{\omega}^{*}\left(\lambda \omega\left(\mathbb{R}^{n}\right)\right) & \leq \limsup _{r \rightarrow \infty}\left(\left(f-m_{f, \omega}(Q(x, r))\right) \chi_{Q(x, r)}\right)_{\omega}^{*}(\lambda \omega(Q(x, r))) \\
& +\limsup _{r \rightarrow \infty}\left(\left(f-m_{f, \omega}(Q(x, r))\right) \chi_{Q(x, r)}\right)_{\omega}^{*}\left(\omega\left(\mathbb{R}^{n}\right) / 4\right) \\
& \leq 2 \limsup _{r \rightarrow \infty}\left(\left(f-m_{f, \omega}(Q(x, r))\right) \chi_{Q(x, r)}\right)_{\omega}^{*}(\lambda \omega(Q(x, r))) \\
& \leq 4 \limsup _{c} \inf _{c}\left((f-c) \chi_{Q(x, r)}\right)_{\omega}^{*}(\lambda \omega(Q(x, r))) \\
& \leq 4 \widetilde{M}_{\lambda, \omega}^{\#} f(x),
\end{aligned}
$$

which finishes the proof.

Median values play an important role in proving the right-hand side of (1.3). In particular, the proof is based on a somewhat stronger variant of the John-Nirenberg inequality: for any cube $Q \subset Q_{0}$ (cf. [Str]),

$$
\left(\left(f-m_{f}(Q)\right) \chi_{Q}\right)^{*}(t) \leq c_{n}\left\|M_{1 / 2 ; Q_{0}}^{\#} f\right\|_{\infty} \log \frac{2|Q|}{t} \quad(0<t<|Q|) .
$$

For any measurable function $f$ define the maximal function $m_{\lambda} f$ by

$$
m_{\lambda} f(x)=\sup _{Q \ni x}\left(f \chi_{Q}\right)^{*}(\lambda|Q|),
$$

where the supremum is taken over all cubes containing $x$. 
Observe that $\left\{x: m_{\lambda} f(x)>\alpha\right\} \subset\left\{x: M \chi_{\{|f|>\alpha\}}(x) \geq \lambda\right\}$. Therefore, by the weak type $(1,1)$ property of the Hardy-Littlewood maximal function, we have

$$
\left|\left\{x: m_{\lambda} f(x)>\alpha\right\}\right| \leq \frac{3^{n}}{\lambda}|\{x:|f(x)|>\alpha\}|
$$

or, equivalently,

$$
\left(m_{\lambda} f\right)^{*}(t) \leq f^{*}\left(\lambda t / 3^{n}\right) \quad(t>0)
$$

We will also need the following lemmas.

Lemma 2.3. For any measurable function $f$, any weight $\omega$, and each cube $Q$,

$$
\left(f \chi_{Q}\right)_{\omega}^{*}(\lambda \omega(Q)) \leq 2 \inf _{c \in \mathbb{R}}\left((f-c) \chi_{Q}\right)_{\omega}^{*}(\lambda \omega(Q))+\left(f \chi_{Q}\right)_{\omega}^{*}((1-\lambda) \omega(Q)),
$$

where $0<\lambda<1$.

This lemma was proved in [L1].

Lemma 2.4. For any $f \in L(Q)$,

$$
\int_{Q}\left|f(x)-f_{Q}\right| d x \leq 8 \int_{Q} M_{\lambda_{n} ; Q}^{\#} f(x) d x .
$$

This lemma contains in [JT], [L3]. It follows easily from (1.6) with $\omega \equiv 1$.

2.3. $A_{\infty}$-weights and $A_{\infty}$-maximal functions. We say that a weight $\omega$ satisfies $A_{\infty}$ Muckenhoupt's condition if there are positive constants $\alpha, \beta<1$ such that $\omega(E) / \omega(Q) \geq \alpha$ implies $|E| /|Q| \geq \beta$ for any cube $Q$ and any subset $E \subset Q$. There are many equivalent characterizations of $A_{\infty}$ (see, e.g., [CF1] or [St, Ch. 5]). In particular, $A_{\infty}$ is equivalent to saying that for any $\alpha^{\prime}, 0<\alpha^{\prime}<1$, there exists a $\beta^{\prime}, 0<\beta^{\prime}<1$, so that $\omega(E) / \omega(Q) \geq \alpha^{\prime}$ implies $|E| /|Q| \geq \beta^{\prime}$ for any $Q$ and $E \subset Q$.

We now, following Wilson [W1], define the maximal function $P_{\lambda} \omega$, which measures a local un- $A_{\infty}$ behaviour of $\omega$. For $0<\lambda<1$ and any cube $Q$ with $\omega(Q)>0$, let $E_{\lambda} \subset Q$ be any subset of minimal Lebesgue measure such that $\omega\left(E_{\lambda}\right)=\lambda \omega(Q)$. Set

$$
P_{\lambda} \omega(x)=\sup _{Q \ni x} \log \left(1+|Q| /\left|E_{\lambda}\right|\right),
$$

where the supremum is taken over all cubes $Q$ with $\omega(Q)>0$ containing $x$.

It is easy to see that $\omega \in A_{\infty}$ if and only if $P_{\lambda} \omega \in L^{\infty}$. We give here several estimates for $P_{\lambda} \omega$. Let $E$ be any subset of $Q$ such that $\omega(E)=\lambda \omega(Q)$. Then, by (2.3),$\lambda \omega(Q) \leq \int_{0}^{|E|}\left(\omega \chi_{Q}\right)^{*}(\tau) d \tau$, and therefore,

$$
\begin{aligned}
\log (1+|Q| /|E|) & \leq \frac{\int_{0}^{|E|}\left(\omega \chi_{Q}\right)^{*}(\tau) \log (1+|Q| / \tau) d \tau}{\int_{0}^{|E|}\left(\omega \chi_{Q}\right)^{*}(\tau) d \tau} \\
& \leq \frac{1}{\lambda \omega(Q)} \int_{0}^{|Q|}\left(\omega \chi_{Q}\right)^{*}(\tau) \log (1+|Q| / \tau) d \tau
\end{aligned}
$$

By a Stein-Herz type inequality (cf. [BS, p. 122]),

$$
\int_{0}^{|Q|}\left(\omega \chi_{Q}\right)^{*}(\tau) \log (1+|Q| / \tau) d \tau \leq 2 \int_{0}^{|Q|}\left(\omega \chi_{Q}\right)^{* *}(\tau) d \tau \leq c_{n} \int_{Q} M_{Q} \omega(x) d x
$$


where $M_{Q} \omega$ is the Hardy-Littlewood maximal function relative to $Q$. Thus, for all $x$,

$$
P_{\lambda} \omega(x) \leq c_{\lambda, n} \sup _{Q \ni x} \frac{1}{\omega(Q)} \int_{Q} M_{Q} \omega(y) d y .
$$

Similarly, one can get the following: for any Young's function $\Phi$ (cf. [BS, p. 265]),

$$
P_{\lambda} \omega(x) \leq \sup _{Q \ni x} \Phi^{-1}\left(\frac{\int_{0}^{|Q|}\left(\omega \chi_{Q}\right)^{*}(\tau) \Phi(\log (1+|Q| / \tau)) d \tau}{\lambda \omega(Q)}\right) .
$$

\section{A weighted Variant of the Bennett-DeVore-Sharpley inequality}

We start with a local analogue of (1.8).

Theorem 3.1. For any $f \in L(Q)$ and all weights $\omega$,

$$
\int_{Q}\left|f(x)-f_{Q}\right| \omega(x) d x \leq c_{n} \int_{Q} M_{\lambda_{n} ; Q}^{\#} f(x) M_{Q} \omega(x) d x .
$$

Proof. The proof follows the same lines as the one of (1.8) in [L3], although with some minor modifications. Clearly, we can assume that $f_{Q}=0$. Suppose also that $2^{l-1} \leq \omega_{Q}<2^{l}$ and $\omega \leq 2^{m}$. If $m-1 \leq l$, then we trivially get, by Lemma 2.4

$$
\begin{aligned}
\int_{Q}|f(x)| \omega(x) d x \leq 4 \omega_{Q} \int_{Q}|f(x)| d x & \leq 32 \inf _{Q} M_{Q} \omega \int_{Q} M_{\lambda_{n} ; Q}^{\#} f(x) d x \\
& \leq 32 \int_{Q} M_{\lambda_{n} ; Q}^{\#} f(x) M_{Q} \omega(x) d x .
\end{aligned}
$$

Assume, therefore, that $l<m-1$. For $l \leq k \leq m-1$ we write $\Omega_{k}=\{x \in Q$ : $\left.M_{Q}^{\Delta} \omega(x)>2^{k}\right\}$ as a disjoint union of dyadic cubes $Q_{j}^{k}$ (relative to $Q$ ) such that $2^{k}<\omega_{Q_{j}^{k}} \leq 2^{n} 2^{k}$ (cf. [St, p. 150]), where $M_{Q}^{\Delta}$ is the dyadic maximal function with respect to $Q$. Now set $g_{k}=\sum_{j}\left(\omega-\omega_{Q_{j}^{k}}\right) \chi_{Q_{j}^{k}}$ and $h_{k}=\omega-g_{k}$. Then $\left|g_{k}-g_{k+1}\right| \leq 3 \cdot 2^{n} 2^{k}$, and $\int_{Q_{j}^{k}}\left(g_{k}-g_{k+1}\right)=0$. Hence, applying Lemma 2.4 and using the fact that $M_{\lambda ; Q}^{\#}|f| \leq M_{\lambda ; Q}^{\#} f$, we get

$$
\begin{aligned}
\int_{Q}|f| \omega d x & =\sum_{k=l}^{m-1} \sum_{j} \int_{Q_{j}^{k}}|f|\left(g_{k}-g_{k+1}\right) d x+\int_{Q}|f| h_{l} d x \\
& \leq \sum_{k=l}^{m-1} \sum_{j} \int_{Q_{j}^{k}}\left(|f|-|f|_{Q_{j}^{k}}\right)\left(g_{k}-g_{k+1}\right) d x+2 \omega_{Q} \int_{Q}|f| d x \\
& \leq\left. 3 \cdot 2^{n} \sum_{k=l}^{m-1} 2^{k} \sum_{j} \int_{Q_{j}^{k}}|| f|-| f\right|_{Q_{j}^{k}} \mid d x+16 \inf _{Q} M_{Q} \omega \int_{Q} M_{\lambda_{n} ; Q}^{\#} f(x) d x \\
& \leq 24 \cdot 2^{n} \sum_{k=l}^{m-1} 2^{k} \sum_{j} \int_{Q_{j}^{k}} M_{\lambda_{n} ; Q}^{\#} f d x+16 \int_{Q} M_{\lambda_{n} ; Q}^{\#} f(x) M_{Q} \omega(x) d x \\
& =24 \cdot 2^{n} \sum_{k=l}^{m-1} 2^{k} \int_{\left\{M_{Q} \omega>2^{k}\right\}} M_{\lambda_{n} ; Q}^{\#} f d x+16 \int_{Q} M_{\lambda_{n} ; Q}^{\#} f(x) M_{Q} \omega(x) d x \\
& \leq 64 \cdot 2^{n} \int_{Q}^{M_{\lambda_{n} ; Q}} f(x) M_{Q} \omega(x) d x .
\end{aligned}
$$


The restriction $\omega \leq 2^{m}$ is easily removed by the Fatou convergence theorem, which completes the proof.

The following covering lemma was proved in [MMNO].

Lemma 3.2. Let $E$ be a subset of $Q$, and suppose that $\omega(E) \leq \rho \omega(Q), 0<\rho<1$. Then there exists a sequence $\left\{Q_{i}\right\}$ of cubes contained in $Q$ such that

(i) $\omega\left(Q_{i} \cap E\right)=\rho \omega\left(Q_{i}\right)$;

(ii) $\bigcup_{i} Q_{i}=\bigcup_{k=1}^{B_{n}} \bigcup_{i \in F_{k}} Q_{i}$, where each of the family $\left\{Q_{i}\right\}_{i \in F_{k}}$ is formed by pairwise disjoint cubes and a constant $B_{n}$ depends only on $n$; in other words, the family $\left\{Q_{i}\right\}$ is almost disjoint with constant $B_{n}$;

(iii) $E^{\prime} \subset \bigcup_{i} Q_{i}$, where $E^{\prime}$ is the set of $\omega$-density points of $E$.

Proof of Theorem 1.1. Since $M_{\lambda ; Q}^{\#}|f| \leq M_{\lambda ; Q}^{\#} f$, we can assume that $f \geq 0$. Applying Lemma 3.2 to the set $E=\left\{x \in Q: f(x)>\left(f \chi_{Q}\right)_{\omega}^{*}(t)\right\}$ and number $\rho=1 / 2$, we get a sequence $\left\{Q_{j}\right\}$ of cubes, for which properties (i), (ii), and (iii) of the lemma hold. By (iii),

$$
\begin{aligned}
t\left(\left(f \chi_{Q}\right)_{\omega}^{* *}(t)-\left(f \chi_{Q}\right)_{\omega}^{*}(t)\right) & =\int_{E}\left(f(x)-\left(f \chi_{Q}\right)^{*}(t)\right) \omega(x) d x \\
& \leq \sum_{j} \int_{E \cap Q_{j}}\left(f(x)-\left(f \chi_{Q}\right)^{*}(t)\right) \omega(x) d x \\
& \leq \sum_{j} \int_{Q_{j}}\left|f(x)-f_{Q_{j}}\right| \omega(x) d x \\
& +\sum_{j} \omega\left(E \cap Q_{j}\right)\left(f_{Q_{j}}-\left(f \chi_{Q}\right)_{\omega}^{*}(t)\right) .
\end{aligned}
$$

We can assume that the last sum is taken over such $j$ for which $f_{Q_{j}}>\left(f \chi_{Q}\right)_{\omega}^{*}(t)$. Since $\omega\left(E \cap Q_{j}\right)=\omega\left(Q_{j}\right) / 2=\omega\left(E^{c} \cap Q_{j}\right)$, we obtain

$$
\omega\left(E \cap Q_{j}\right)\left(f_{Q_{j}}-\left(f \chi_{Q}\right)_{\omega}^{*}(t)\right) \leq \int_{E^{c} \cap Q_{j}}\left(f_{Q_{j}}-f(\xi)\right) \omega(\xi) d \xi,
$$

and, therefore,

$$
t\left(\left(f \chi_{Q}\right)_{\omega}^{* *}(t)-\left(f \chi_{Q}\right)_{\omega}^{*}(t)\right) \leq 2 \sum_{j} \int_{Q_{j}}\left|f(x)-f_{Q_{j}}\right| \omega(x) d x .
$$

Now using properties (i), (ii) of Lemma 3.2, Theorem 3.1, and (2.3), we have

$$
\begin{aligned}
\sum_{j} \int_{Q_{j}}\left|f(x)-f_{Q_{j}}\right| \omega(x) d x & \leq c_{n} \sum_{j} \int_{Q_{j}} M_{\lambda_{n} ; Q}^{\#} f(x) M_{Q} \omega(x) d x \\
& \leq c_{n} \sum_{k=1}^{B_{n}} \sum_{j \in F_{k}} \int_{Q_{j}} M_{\lambda_{n} ; Q}^{\#} f(x) M_{Q} \omega(x) d x \\
& \leq c_{n} \sum_{k=1}^{B_{n}} \int_{0}^{\omega\left(\cup_{j \in F_{k}} Q_{j}\right)}\left(\left(M_{\lambda_{n} ; Q}^{\#} f M_{Q} \omega / \omega\right) \chi_{Q}\right)_{\omega}^{*}(\tau) d \tau \\
& \leq c_{n} B_{n} \int_{0}^{2 t}\left(\left(M_{\lambda_{n} ; Q}^{\#} f M_{Q} \omega / \omega\right) \chi_{Q}\right)_{\omega}^{*}(\tau) d \tau
\end{aligned}
$$


(here we also used that $\omega(E) \leq t$, and so, $\left.\omega\left(\bigcup_{j \in F_{k}} Q_{j}\right) \leq 2 \omega(E) \leq 2 t\right)$. Hence,

$$
\begin{aligned}
\left(f \chi_{Q}\right)_{\omega}^{* *}(t)-\left(f \chi_{Q}\right)_{\omega}^{*}(t) & \leq 4 c_{n} B_{n}\left(\left(M_{\lambda_{n} ; Q}^{\#} f M_{Q} \omega / \omega\right) \chi_{Q}\right)_{\omega}^{* *}(2 t) \\
& \leq 4 c_{n} B_{n}\left(\left(M_{\lambda_{n} ; Q}^{\#} f M_{Q} \omega / \omega\right) \chi_{Q}\right)_{\omega}^{* *}(t),
\end{aligned}
$$

as required.

Remark 3.3. If $\omega$ is Lebesgue measure, then $f_{Q}^{\#} \asymp M_{Q} M_{\lambda_{n} ; Q}^{\#} f$ (see [JT]), and $\left(f \chi_{Q}\right)^{* *}(t) \asymp\left(M_{Q} f\right)^{*}(t)$ (see, e.g., [BS] p. 122]). Hence, $\left(M_{\lambda_{n} ; Q}^{\#} f\right)^{* *}(t) \asymp\left(f_{Q}^{\#}\right)^{*}(t)$, and we obtain that in this case Theorem 1.1 is equivalent to the Bennett-DeVoreSharpley theorem (cf. (1.5)).

Corollary 3.4. For any measurable $f$ on $\mathbb{R}^{n}$ with $f^{*}(\infty)=0$, and any weight $\omega$,

$$
f_{\omega}^{* *}(t) \leq c_{n} \int_{t}^{\omega\left(\mathbb{R}^{n}\right)}\left(M_{\lambda_{n}}^{\#} f M \omega / \omega\right)_{\omega}^{* *}(s) \frac{d s}{s} \quad\left(0<t<\omega\left(\mathbb{R}^{n}\right)\right) .
$$

Proof. Integrating (1.11) gives

$$
\begin{aligned}
\left(f \chi_{Q}\right)_{\omega}^{* *}(t) & =\int_{t}^{\omega(Q) / 2}\left(\left(f \chi_{Q}\right)_{\omega}^{* *}(s)-\left(f \chi_{Q}\right)_{\omega}^{*}(s)\right) \frac{d s}{s}+\left(f \chi_{Q}\right)_{\omega}^{* *}(\omega(Q) / 2) \\
& \leq c_{n} \int_{t}^{\omega(Q)}\left(\left(M_{\lambda_{n} ; Q}^{\#} f \frac{M_{Q} \omega}{\omega}\right) \chi_{Q}\right)_{\omega}^{* *}(s) \frac{d s}{s}+\frac{2}{\omega(Q)} \int_{Q}|f| \omega d x,
\end{aligned}
$$

provided $0<t<\omega(Q)$. Next, it follows from the properties of median values (cf. Section 2.2), from Lemma 2.4 and Theorem 3.1 that

$$
\begin{aligned}
\frac{1}{\omega(Q)} \int_{Q}|f| \omega & \leq \frac{1}{\omega(Q)} \int_{Q}\left|f-f_{Q}\right| \omega d x+\left|f_{Q}-m_{f}(Q)\right|+\left|m_{f}(Q)\right| \\
& \leq \frac{1}{\omega(Q)} \int_{Q}\left|f-f_{Q}\right| \omega d x+\frac{2}{|Q|} \int_{Q}\left|f-f_{Q}\right| d x+\left(f \chi_{Q}\right)^{*}(|Q| / 2) \\
& \leq \frac{c_{n}}{\omega(Q)} \int_{Q} M_{\lambda_{n} ; Q}^{\#} f M_{Q} \omega d x+\frac{16}{|Q|} \int_{Q} M_{\lambda_{n} ; Q}^{\#} f d x+\left(f \chi_{Q}\right)^{*}(|Q| / 2) \\
& \leq \frac{c_{n}+16}{\omega(Q)} \int_{Q} M_{\lambda_{n} ; Q}^{\#} f M_{Q} \omega d x+\left(f \chi_{Q}\right)^{*}(|Q| / 2) \\
& \leq c_{n}^{\prime} \int_{\omega(Q) / 2}^{\omega(Q)}\left(\left(M_{\lambda_{n} ; Q}^{\#} f M_{Q} \omega / \omega\right) \chi_{Q}\right)^{* *}(s) \frac{d s}{s}+\left(f \chi_{Q}\right)^{*}(|Q| / 2) .
\end{aligned}
$$

From this and from the previous estimate we obtain

$$
\begin{aligned}
\left(f \chi_{Q}\right)_{\omega}^{* *}(t) & \leq c_{n} \int_{t}^{\omega(Q)}\left(\left(M_{\lambda_{n} ; Q}^{\#} f M_{Q} \omega / \omega\right) \chi_{Q}\right)^{* *}(s) \frac{d s}{s}+2\left(f \chi_{Q}\right)^{*}(|Q| / 2) \\
& \leq c_{n} \int_{t}^{\omega\left(\mathbb{R}^{n}\right)}\left(M_{\lambda_{n}}^{\#} f M \omega / \omega\right)_{\omega}^{* *}(s) \frac{d s}{s}+2 f^{*}(|Q| / 2) .
\end{aligned}
$$

Here letting $Q \rightarrow \mathbb{R}^{n}$ and using (2.2), we get (3.1).

Now we can easily prove (1.9).

Corollary 3.5. For any measurable $f$ with $f^{*}(\infty)=0$, and any weight $\omega$,

$$
\int_{\mathbb{R}^{n}}|f|^{p} \omega d x \leq c_{p, n} \int_{\mathbb{R}^{n}}\left(M_{\lambda_{n}}^{\#} f\right)^{p}(M \omega / \omega)^{p} \omega d x \quad(1 \leq p<\infty) .
$$


Proof. In the case $p=1$ we trivially obtain from (3.1) that

$$
\int_{0}^{t} f_{\omega}^{*}(\tau) d \tau \leq c_{n} t\left\|M_{\lambda_{n}}^{\#} f M \omega / \omega\right\|_{L_{\omega}^{1}} \int_{t}^{\infty} \frac{d s}{s^{2}}=c_{n}\left\|M_{\lambda_{n}}^{\#} f M \omega\right\|_{L^{1}} .
$$

Here letting $t \rightarrow \omega\left(\mathbb{R}^{n}\right)$ yields (3.2).

Suppose $p>1$. Then we apply (3.1) and Hardy's inequalities [BS, p. 124]:

$$
\begin{aligned}
\|f\|_{L_{\omega}^{p}} & =\left\|f_{\omega}^{*}\right\|_{L^{p}\left(0, \omega\left(\mathbb{R}^{n}\right)\right)} \leq\left\|f_{\omega}^{* *}\right\|_{L^{p}\left(0, \omega\left(\mathbb{R}^{n}\right)\right)} \\
& \leq c_{n} p\left\|\left(M_{\lambda_{n}}^{\#} f M \omega / \omega\right)_{\omega}^{* *}\right\|_{L^{p}\left(0, \omega\left(\mathbb{R}^{n}\right)\right)} \\
& \leq c_{n} \frac{p^{2}}{p-1}\left\|\left(M_{\lambda_{n}}^{\#} f M \omega / \omega\right)_{\omega}^{*}\right\|_{L^{p}\left(0, \omega\left(\mathbb{R}^{n}\right)\right)}=c_{n} \frac{p^{2}}{p-1}\left\|M_{\lambda_{n}}^{\#} f M \omega / \omega\right\|_{L_{\omega}^{p}},
\end{aligned}
$$

and we are done.

Also we obtain a new weak-type estimate for singular integrals.

Corollary 3.6. For any $f \in \bigcup_{p \geq 1} L^{p}$, and any weight $\omega$,

$$
(T f)_{\omega}^{* *}(t) \leq c_{n} \int_{t}^{\omega\left(\mathbb{R}^{n}\right)}(\operatorname{MfM} \omega / \omega)_{\omega}^{* *}(s) \frac{d s}{s} \quad\left(0<t<\omega\left(\mathbb{R}^{n}\right)\right) .
$$

Proof. Since

$$
f \in \bigcup_{p \geq 1} L^{p} \Rightarrow(T f) \in\left(\operatorname{weak} L^{1}\right) \cup \bigcup_{p>1} L^{p} \Rightarrow(T f)^{*}(\infty)=0,
$$

we can apply (1.4) and (3.1), which immediately gives the required estimate.

\section{A SElF-IMPROVInG PROPERTY OF SharP FUnCtion INEQUALITIES}

It was observed in [L3] that a known pointwise estimate [L1]

$$
M_{\lambda}^{\#}(M f)(x) \leq c_{\lambda, n} f^{\#}(x)
$$

combined with (11.9) and (1.10) immediately yields the following weighted versions of the Fefferman-Stein theorem (cf. [FS2]):

$$
\int_{\mathbb{R}^{n}}(M f)^{p} \omega d x \leq c_{p, n} \int_{\mathbb{R}^{n}}\left(f^{\#}\right)^{p}(M \omega / \omega)^{p} \omega d x \quad(1 \leq p<\infty)
$$

and

$$
\int_{\mathbb{R}^{n}}(M f)^{p} \omega d x \leq c_{p, n} \int_{\mathbb{R}^{n}}\left(f^{\#}\right)^{p} M^{[p]+1} \omega d x \quad(1<p<\infty) .
$$

Note also that it follows from (1.8), by Chebyshev inequality,

$$
f_{\omega}^{*}(t) \leq \frac{c_{n}}{t} \int_{\mathbb{R}^{n}} M_{\lambda_{n}}^{\#} f(x) M \omega(x) d x .
$$

In this section we show that inequalities (1.9), (1.10) (as well as (4.1), (4.2)) for $p>1$, and (4.3) can be improved.

Theorem 4.1. For any locally integrable $f$ with $f^{*}(\infty)=0$, and any weight $\omega$,

$$
\begin{gathered}
\int_{\mathbb{R}^{n}}(M f)^{p} \omega d x \leq c_{p, n} \int_{\mathbb{R}^{n}}\left(M_{\lambda_{n}}^{\#} f\right)^{p}(M \omega / \omega)^{p} \omega d x \quad(1<p<\infty), \\
\int_{\mathbb{R}^{n}}(M f)^{p} \omega d x \leq c_{p, n} \int_{\mathbb{R}^{n}}\left(M_{\lambda_{n}}^{\#} f\right)^{p} M^{[p]+1} \omega d x \quad(1<p<\infty),
\end{gathered}
$$


and

$$
(M f)_{\omega}^{*}(t) \leq \frac{c_{n}}{t} \int_{\mathbb{R}^{n}} M_{\lambda_{n}}^{\#} f(x) M \omega(x) d x \quad(t>0) .
$$

It is interesting that the proof of the theorem is essentially based on inequalities (1.9), (1.10), and (4.3), that is, these inequalities have a self-improving property expressed in (4.4), (4.5), and (4.6), respectively. An important ingredient of the proof is also the following classical Fefferman-Stein inequalities [FS1]:

$$
\int_{\mathbb{R}^{n}}(M f)^{p} \omega d x \leq c_{p, n} \int_{\mathbb{R}^{n}}|f|^{p} M \omega d x \quad(1<p<\infty),
$$

and

$$
(M f)_{\omega}^{*}(t) \leq \frac{c_{n}}{t} \int_{\mathbb{R}^{n}}|f| M \omega d x \quad(t>0) .
$$

Note, however, that a direct combination of, for instance, (4.7) and (1.10), yields only an inequality like (4.5) with $M^{[p]+2} \omega$ in place of $M^{[p]+1} \omega$ on the right-hand side. To prove the theorem, we will need several following pointwise inequalities.

Proposition 4.2. For any locally integrable $f$ and all $x$,

$$
\begin{gathered}
M f(x) \leq 3 f^{\#}(x)+m_{1 / 2} f(x), \\
f^{\#}(x) \leq 8 M M_{\lambda_{n}}^{\#} f(x),
\end{gathered}
$$

and

$$
M_{\lambda}^{\#}\left(m_{1 / 2} f\right)(x) \leq 4 M_{\lambda / 2 \cdot 9^{n}}^{\#} f(x) .
$$

Proof of Theorem 4.1. By (4.9), (4.10) and Minkowski's inequality,

$$
\|M f\|_{p, \omega} \leq 24\left\|M M_{\lambda_{n}}^{\#} f\right\|_{p, \omega}+\left\|m_{1 / 2} f\right\|_{p, \omega} .
$$

To estimate the first term on the right-hand side we use 4.7, while to estimate the second one we apply (1.9) and (4.11) (observing that, by (2.7), the condition $f^{*}(\infty)=0$ implies $\left.\left(m_{1 / 2} f\right)^{*}(\infty)=0\right)$. So,

$$
\int_{\mathbb{R}^{n}}\left(M M_{\lambda_{n}}^{\#} f\right)^{p} \omega d x \leq c \int_{\mathbb{R}^{n}}\left(M_{\lambda_{n}}^{\#} f\right)^{p} M \omega d x,
$$

and

$$
\begin{aligned}
\int_{\mathbb{R}^{n}}\left(m_{1 / 2} f\right)^{p} \omega d x & \leq c \int_{\mathbb{R}^{n}}\left(M_{\lambda_{n}}^{\#}\left(m_{1 / 2} f\right)\right)^{p}(M \omega / \omega)^{p} \omega d x \\
& \leq c^{\prime} \int_{\mathbb{R}^{n}}\left(M_{\lambda_{n}^{\prime}}^{\#} f\right)^{p}(M \omega / \omega)^{p} \omega d x .
\end{aligned}
$$

Since $M \omega \leq(M \omega / \omega)^{p} \omega$, we obtain 4.4 . The proof of 4.5 is exactly the same, only (1.10) should be applied instead of (1.9). The proof of (4.6) also follows the same lines with some minor modifications. Namely, to get (4.6) we apply a subadditivity property of rearrangements (2.1) instead of Minkowski's inequality, and then we use (4.3) and (4.8). 
Proof of Proposition 4.2. For any cube $Q$ containing $x$ and any constant $c$,

$$
\begin{aligned}
\frac{1}{|Q|} \int_{Q}|f(x)| d x & \leq \frac{1}{|Q|} \int_{Q}|f(x)-c| d x+\left|m_{f}(Q)-c\right|+\left|m_{f}(Q)\right| \\
& \leq \frac{3}{|Q|} \int_{Q}|f(x)-c| d x+\left|m_{f}(Q)\right|,
\end{aligned}
$$

which proves 4.9). Next, (4.10) is an immediate corollary of Lemma 2.4 (this inequality also contains in $[\mathrm{JT}]$ ).

We now prove (4.11). Let $Q$ be any cube containing $x$. Take an arbitrary point $y \in Q$, and let $Q^{\prime}$ be any cube containing $y$. If $Q^{\prime} \subset 3 Q$, then

$$
\begin{aligned}
\left(f \chi_{Q^{\prime}}\right)^{*}\left(\left|Q^{\prime}\right| / 2\right) & \leq\left(\left(f-m_{f}(3 Q)\right) \chi_{Q^{\prime}}\right)^{*}\left(\left|Q^{\prime}\right| / 2\right)+\left|m_{f}(3 Q)\right| \\
& \leq m_{1 / 2}\left(\left(f-m_{f}(3 Q)\right) \chi_{3 Q}\right)(y)+\inf _{\xi \in Q} m_{1 / 2} f(\xi) .
\end{aligned}
$$

Assume that $Q^{\prime} \not \subset 3 Q$. Then $Q \subset 3 Q^{\prime}$ and in this case we apply Lemma 2.3 to get

$$
\begin{aligned}
& \left(f \chi_{Q^{\prime}}\right)^{*}\left(\left|Q^{\prime}\right| / 2\right) \leq\left(f \chi_{3 Q^{\prime}}\right)^{*}\left(\left|3 Q^{\prime}\right| / 2 \cdot 3^{n}\right) \\
& \leq 2 \inf _{c}\left((f-c) \chi_{3 Q^{\prime}}\right)^{*}\left(\left|3 Q^{\prime}\right| / 2 \cdot 3^{n}\right)+\left(f \chi_{3 Q^{\prime}}\right)^{*}\left(\left(1-1 / 2 \cdot 3^{n}\right)\left|3 Q^{\prime}\right|\right) \\
& \leq 2 M_{1 / 2 \cdot 3^{n}}^{\#} f(x)+\inf _{\xi \in Q} m_{1 / 2} f(\xi) .
\end{aligned}
$$

Therefore, for all $y \in Q$,

$$
\begin{aligned}
m_{1 / 2} f(y) & =\max \left(\sup _{Q^{\prime} \ni y, Q^{\prime} \subset 3 Q}\left(f \chi_{Q^{\prime}}\right)^{*}\left(\left|Q^{\prime}\right| / 2\right), \sup _{Q^{\prime} \ni y, Q \subset 3 Q^{\prime}}\left(f \chi_{Q^{\prime}}\right)^{*}\left(\left|Q^{\prime}\right| / 2\right)\right) \\
& \leq m_{1 / 2}\left(\left(f-m_{f}(3 Q)\right) \chi_{3 Q}\right)(y)+2 M_{1 / 2 \cdot 3^{n}}^{\#} f(x)+\inf _{\xi \in Q} m_{1 / 2} f(\xi) .
\end{aligned}
$$

Hence, applying (2.7) yields

$$
\begin{aligned}
& \left(\left(m_{1 / 2} f-\inf _{Q} m_{1 / 2} f\right) \chi_{Q}\right)^{*}(\lambda|Q|) \\
& \leq\left(m_{1 / 2}\left(\left(f-m_{f}(3 Q)\right) \chi_{3 Q}\right)\right)^{*}(\lambda|Q|)+2 M_{1 / 2 \cdot 3^{n}}^{\#} f(x) \\
& \leq\left(\left(f-m_{f}(3 Q)\right) \chi_{3 Q}\right)^{*}\left(\lambda|3 Q| / 2 \cdot 9^{n}\right)+2 M_{1 / 2 \cdot 3^{n}}^{\#} f(x) \leq 4 M_{\lambda / 2 \cdot 9^{n}}^{\#} f(x),
\end{aligned}
$$

which gives (4.11).

\section{Some estimates For the Centered local SharP FUnCtion}

In Section 3 we have obtained a weighted rearrangement inequality for the unweighted local sharp function. Here we prove an inequality of different type, namely, a weighted rearrangement inequality for the centered weighted local sharp function $\widehat{M}_{\lambda, \omega}^{\#} f$.

Proof of Theorem 1.2. The proof is a modification of the method used in proving (1.6) (cf. [L1], [L2]). Set $\Omega=\left\{x: \widetilde{M}_{\lambda_{n}, \omega}^{\#} f(x)>\left(\widetilde{M}_{\lambda_{n}, \omega}^{\#} f\right)_{\omega}^{*}(t / 2)\right\}$, where $\lambda_{n}<1$ is some constant depending only on $n$ which will be chosen later. Let $E$ be an arbitrary set with $\omega(E)=t$. Choose a compact subset $\widetilde{E} \subset E$ with $\omega(\widetilde{E}) \geq 9 t / 10$. Clearly, $\omega(\widetilde{E} \backslash \Omega) \geq 2 t / 5$. Next, for almost every point $x \in \widetilde{E} \backslash \Omega$ there is a cube $Q_{x}$ centered at $x$ and such that $\omega\left((\widetilde{E} \backslash \Omega) \cap Q_{x}\right)=\lambda_{n} \omega\left(Q_{x}\right)$. Applying the Besicovitch Covering Theorem to the family $\left\{Q_{x}\right\}_{x \in \widetilde{E} \backslash \Omega}$ yields a countable collection of cubes $Q_{j}$, covering $\widetilde{E} \backslash \Omega$, and such that they are almost disjoint with 
constant $B_{n}$, that is, $\bigcup_{j} Q_{j}=\bigcup_{k=1}^{B_{n}} \bigcup_{j \in F_{k}} Q_{j}$, where each of the family $\left\{Q_{j}\right\}_{j \in F_{k}}$ is formed by pairwise disjoint cubes. Then

$$
2 t / 5 \leq \sum_{k=1}^{B_{n}} \sum_{j \in F_{k}} \omega\left((\widetilde{E} \backslash \Omega) \cap Q_{j}\right)=\lambda_{n} \sum_{k=1}^{B_{n}} \sum_{j \in F_{k}} \omega\left(Q_{j}\right),
$$

and hence, for some $k_{0}$,

$$
2 t / 5 \lambda_{n} B_{n} \leq \sum_{j \in F_{k_{0}}} \omega\left(Q_{j}\right) .
$$

Next, since the centers of $Q_{j}$ lie outside $\Omega$,

$$
\inf _{c}\left((f-c) \chi_{Q_{j}}\right)_{\omega}^{*}\left(\lambda_{n} \omega\left(Q_{j}\right)\right) \leq\left(\widetilde{M}_{\lambda_{n}, \omega}^{\#} f\right)_{\omega}^{*}(t / 2) .
$$

From this and from Lemma 2.3 we get

$$
\begin{aligned}
\inf _{x \in E}|f(x)| & \leq \inf _{j \in F_{k_{0}}} \inf _{x \in E \cap Q_{j}}|f(x)| \leq \inf _{j \in F_{k_{0}}}\left(f \chi_{Q_{j}}\right)_{\omega}^{*}\left(\lambda_{n} \omega\left(Q_{j}\right)\right) \\
& \leq 2\left(\widetilde{M}_{\lambda_{n}, \omega}^{\#} f\right)_{\omega}^{*}(t / 2)+\inf _{j \in F_{k_{0}}}\left(f \chi_{Q_{j}}\right)_{\omega}^{*}\left(\left(1-\lambda_{n}\right) \omega\left(Q_{j}\right)\right) .
\end{aligned}
$$

Since the cubes from $F_{k_{0}}$ are pairwise disjoint, we easily obtain that

$$
\begin{aligned}
\inf _{j \in F_{k_{0}}}\left(f \chi_{Q_{j}}\right)_{\omega}^{*}\left(\left(1-\lambda_{n}\right) \omega\left(Q_{j}\right)\right) & \leq f_{\omega}^{*}\left(\left(1-\lambda_{n}\right) \sum_{j \in F_{k_{0}}} \omega\left(Q_{j}\right)\right) \\
& \leq f_{\omega}^{*}\left(2\left(1-\lambda_{n}\right) t / 5 \lambda_{n} B_{n}\right) .
\end{aligned}
$$

Now choose $\lambda_{n}$ so that $2\left(1-\lambda_{n}\right) / 5 \lambda_{n} B_{n}=2$, that is, $\lambda_{n}=1 /\left(5 B_{n}+1\right)$. Then

$$
\inf _{x \in E}|f(x)| \leq 2\left(\widetilde{M}_{\lambda_{n}, \omega}^{\#} f\right)_{\omega}^{*}(t / 2)+f_{\omega}^{*}(2 t) .
$$

Taking the supremum over all $E$ with $\omega(E)=t$ yields

$$
f_{\omega}^{*}(t) \leq 2\left(\widetilde{M}_{\lambda_{n}, \omega}^{\#} f\right)_{\omega}^{*}(t / 2)+f_{\omega}^{*}(2 t),
$$

and therefore the theorem is proved.

In what follows, we will assume that $m_{f, \omega}$ is a weighted median value of $f$ over $\mathbb{R}^{n}$ if $\omega\left(\mathbb{R}^{n}\right)<\infty$ and $f$ is any measurable function, and $m_{f, \omega}=0$ if $\omega\left(\mathbb{R}^{n}\right)=\infty$ and $f_{\omega}^{*}(\infty)=0$.

Corollary 5.1. If either $\omega\left(\mathbb{R}^{n}\right)<\infty$ or $\omega\left(\mathbb{R}^{n}\right)=\infty$ and $f_{\omega}^{*}(\infty)=0$, then

$$
\left(f-m_{f, \omega}\right)_{\omega}^{*}(t)^{\delta} \leq 6 \int_{t / 4}^{\omega\left(\mathbb{R}^{n}\right)}\left(\widetilde{M}_{\lambda_{n}, \omega}^{\#} f\right)_{\omega}^{*}(s)^{\delta} \frac{d s}{s} \quad\left(0<t<\omega\left(\mathbb{R}^{n}\right)\right),
$$

where $0<\delta \leq 1$, and

$$
\left\|f-m_{f, \omega}\right\|_{L_{\omega}^{p}} \asymp\left\|\widetilde{M}_{\lambda_{n}, \omega}^{\#} f\right\|_{L_{\omega}^{p}} \quad(0<p<\infty) .
$$

Proof. Consider, for example, the case $\omega\left(\mathbb{R}^{n}\right)<\infty$.

The proof of (5.2) is quite standard (cf. [L1]). Iterating (5.1) and using the elementary inequality $(a+b)^{\delta} \leq a^{\delta}+b^{\delta}, a, b \geq 0$, we get

$$
f_{\omega}^{*}(t)^{\delta} \leq 2^{\delta} \int_{t / 4}^{\omega\left(\mathbb{R}^{n}\right)}\left(\widetilde{M}_{\lambda_{n}, \omega}^{\#} f\right)_{\omega}^{*}(s)^{\delta} \frac{d s}{s}+f_{\omega}^{*}\left(\lambda_{n} \omega\left(\mathbb{R}^{n}\right)\right)^{\delta} .
$$


By Proposition 2.2

$$
\begin{aligned}
\left(f-m_{f, \omega}\right)_{\omega}^{*}\left(\lambda_{n} \omega\left(\mathbb{R}^{n}\right)\right)^{\delta} & \leq\left(4 \inf _{x \in \mathbb{R}^{n}} \widetilde{M}_{\lambda_{n}, \omega}^{\#} f(x)\right)^{\delta} \leq 4^{\delta}\left(\widetilde{M}_{\lambda_{n}, \omega}^{\#} f\right)_{\omega}^{*}\left(\omega\left(\mathbb{R}^{n}\right)\right)^{\delta} \\
& \leq \frac{4^{\delta}}{\log 4} \int_{\omega\left(\mathbb{R}^{n}\right) / 4}^{\omega\left(\mathbb{R}^{n}\right)}\left(\widetilde{M}_{\lambda_{n}, \omega}^{\#} f\right)_{\omega}^{*}(s)^{\delta} \frac{d s}{s} .
\end{aligned}
$$

From this and from (5.4) with $f-m_{f, \omega}$ in place of $f$ we obtain (5.2).

Next, (5.2) along with Hardy's inequality [BS] p. 124] immediately gives

$$
\left\|f-m_{f, \omega}\right\|_{L_{\omega}^{p}} \leq c_{p}\left\|\widetilde{M}_{\lambda_{n}, \omega}^{\#} f\right\|_{L_{\omega}^{p}} \quad(0<p<\infty) .
$$

To prove the converse, we define the maximal function $\widetilde{m}_{\lambda, \omega} f$ by

$$
\widetilde{m}_{\lambda, \omega} f(x)=\sup _{Q \ni x}\left(f \chi_{Q}\right)_{\omega}^{*}(\omega(Q)),
$$

where the supremum is taken over all cubes centered at $x$. Now, exactly as in proving (2.7), we get $\left\{\widetilde{m}_{\lambda, \omega} f>\alpha\right\} \subset\left\{\widetilde{M}_{\omega} \chi_{\{|f|>\alpha\}} \geq \lambda\right\}$, and thus,

$$
\omega\left\{\widetilde{m}_{\lambda, \omega} f>\alpha\right\} \leq \frac{c_{n}}{\lambda} \omega\{|f|>\alpha\}
$$

or, equivalently,

$$
\left(\widetilde{m}_{\lambda, \omega} f\right)_{\omega}^{*}(t) \leq f_{\omega}^{*}\left(\lambda t / c_{n}\right)
$$

(here $\widetilde{M}_{\omega}$ is the weighted centered Hardy-Littlewood maximal function). Since $\widetilde{M}_{\lambda_{n}, \omega}^{\#} f \leq \widetilde{m}_{\lambda_{n}, \omega}\left(f-m_{f, \omega}\right)$, we obtain $\left(\widetilde{M}_{\lambda, \omega}^{\#} f\right)_{\omega}^{*}(t) \leq\left(f-m_{f, \omega}\right)_{\omega}^{*}\left(\lambda_{n} t / c_{n}\right)$, so it follows that

$$
\left\|\widetilde{M}_{\lambda_{n}, \omega}^{\#} f\right\|_{L_{\omega}^{p}} \leq\left(c_{n} / \lambda_{n}\right)^{1 / p}\left\|f-m_{f, \omega}\right\|_{L_{\omega}^{p}} \quad(0<p<\infty) .
$$

This concludes the proof of (5.3).

The case when $\omega\left(\mathbb{R}^{n}\right)=\infty$ and $f_{\omega}^{*}(\infty)=0$ is essentially the same. Note only that this case is even simpler, since the second term on the right-hand side of (5.4) will be equal to zero, and (5.3) will follow without using Proposition 2.2

Our aim now is to estimate $\widetilde{M}_{\lambda_{n}, \omega}^{\#} f$ by the unweighted local sharp function. The main tool we will use is the following interpolation lemma, which resembles estimates of $K$-functional for $\left(L^{1}, B M O\right)\left[\mathrm{BS}\right.$, p. 393] and $E$-functional for $\left(L^{0}, B M O\right)$ [JT, Theorem 3.2]. These estimates were proved by means of the Whitney Covering Theorem. A local case of such estimates requires some modifications. For instance, we have to apply a local variant of the Whitney Theorem to an open set $\Omega$ which is strictly contained in $Q$. But for our purposes it will be convenient to use the lemma below with any open set, not necessarily strictly contained in $Q$. To avoid some technical difficulties, we will give a different proof, which perhaps is of some independent interest. Our proof does not use the Whitney Theorem.

Lemma 5.2. Let $f$ be any measurable function defined on a cube $Q_{0}$, and let $\Omega$ be an open subset of $Q$ with $\left|F \equiv Q_{0} \backslash \Omega\right|>0$. Then there is a function $g$ such that $f=g$ on $F$, and

$$
\left\|M_{1 / 2 ; Q_{0}}^{\#} g\right\|_{\infty} \leq \sup _{x \in F} M_{\lambda_{n} ; Q_{0}}^{\#} f(x)
$$


Proof. Let $\mathcal{Q}$ be a collection of cubes $Q$ contained in $\Omega$ and such that $\operatorname{dist}(Q, F)=$ $\operatorname{diam}(Q)$. For $x \in \Omega$ we consider the maximal function $A f$ defined by

$$
A f(x)=\sup _{x \in Q \in \mathcal{Q}} m_{f}(Q),
$$

where the supremum is taken over all cubes $Q \in \mathcal{Q}$ containing $x$. Set now $g=$ $(A f) \chi_{\Omega}+f \chi_{F}$. We have to estimate $\inf _{c}\left((g-c) \chi_{Q^{\prime}}\right)^{*}\left(\left|Q^{\prime}\right| / 2\right)$ for any $Q^{\prime} \subset Q_{0}$. There are only three cases.

Case 1. Suppose $Q^{\prime} \cap \Omega=\emptyset$. This case is trivial, since we have

$$
\inf _{c}\left((g-c) \chi_{Q^{\prime}}\right)^{*}\left(\left|Q^{\prime}\right| / 2\right)=\inf _{c}\left((f-c) \chi_{Q^{\prime}}\right)^{*}\left(\left|Q^{\prime}\right| / 2\right) \leq \sup _{x \in F} M_{1 / 2 ; Q_{0}}^{\#} f(x) .
$$

Case 2. Let $Q^{\prime} \cap \Omega \neq \emptyset$, and suppose for some $y \in Q^{\prime} \cap \Omega$ there is a cube $Q^{*} \in \mathcal{Q}$ containing $y$ and such that $\operatorname{diam}\left(Q^{\prime}\right) \leq \operatorname{diam}\left(Q^{*}\right) / 2$. Then it is clear that $Q^{\prime} \subset \Omega$. Let us show that for any $\bar{Q} \in \mathcal{Q}$ with $\bar{Q} \cap Q^{\prime} \neq \emptyset$,

$$
\frac{1}{4} \operatorname{diam}\left(Q^{*}\right) \leq \operatorname{diam}(\bar{Q}) \leq \frac{5}{2} \operatorname{diam}\left(Q^{*}\right) .
$$

First, we note that $\operatorname{dist}\left(Q^{*}, F\right) \leq \operatorname{dist}\left(Q^{\prime}, F\right)+\operatorname{diam}\left(Q^{\prime}\right)$, which implies

$$
\frac{1}{2} \operatorname{diam}\left(Q^{*}\right) \leq \operatorname{dist}\left(Q^{\prime}, F\right) \text {. }
$$

On the other hand,

$$
\operatorname{dist}\left(Q^{\prime}, F\right) \leq \operatorname{dist}\left(Q^{*}, F\right)+\operatorname{diam}\left(Q^{*}\right) \leq 2 \operatorname{diam}\left(Q^{*}\right) .
$$

Assume that $\operatorname{dist}(\bar{Q}, F) \leq \operatorname{dist}\left(Q^{\prime}, F\right)$. In this case

$$
\operatorname{diam}(\bar{Q}) \leq \operatorname{dist}\left(Q^{\prime}, F\right) \leq \operatorname{dist}(\bar{Q}, F)+\operatorname{diam}(\bar{Q}) \leq 2 \operatorname{diam}(\bar{Q}),
$$

and, by (5.7) and (5.8),

$$
\frac{1}{4} \operatorname{diam}\left(Q^{*}\right) \leq \operatorname{diam}(\bar{Q}) \leq 2 \operatorname{diam}\left(Q^{*}\right) .
$$

If $\operatorname{dist}(\bar{Q}, F)>\operatorname{dist}\left(Q^{\prime}, F\right)$, then again applying (5.7) and (5.8) yields

$$
\begin{aligned}
\frac{1}{2} \operatorname{diam}\left(Q^{*}\right) & \leq \operatorname{dist}\left(Q^{\prime}, F\right)<\operatorname{diam}(\bar{Q}) \\
& =\operatorname{dist}(\bar{Q}, F) \leq \operatorname{dist}\left(Q^{\prime}, F\right)+\operatorname{diam}\left(Q^{\prime}\right) \leq \frac{5}{2} \operatorname{diam}\left(Q^{*}\right) .
\end{aligned}
$$

Unifying (5.9) and (5.10), we get (5.6).

Let $E$ be the union of all cubes $\bar{Q} \in \mathcal{Q}$ with $\bar{Q} \cap Q^{\prime} \neq \emptyset$, and let $\widetilde{Q}$ be a cube of minimal measure containing $E$. Then $\operatorname{dist}(\widetilde{Q}, F)<\operatorname{diam}(\widetilde{Q})$, and, by (5.6),

$$
\frac{1}{4} \operatorname{diam}\left(Q^{*}\right) \leq \operatorname{diam}(\widetilde{Q}) \leq \frac{11}{2} \operatorname{diam}\left(Q^{*}\right)
$$

It follows easily from the properties of $\widetilde{Q}$ that there is a cube $\widetilde{\widetilde{Q}} \subset Q_{0}$ containing $\widetilde{Q}$ and such that $\widetilde{\widetilde{Q}} \cap F \neq \underset{\widetilde{\widetilde{Q}}}{\widetilde{a}}$ and $|\widetilde{\widetilde{Q}}| \leq 3^{n}|\widetilde{Q}|$. We get that any cube $\bar{Q} \in \mathcal{Q}$ with $\bar{Q} \cap Q^{\prime} \neq \emptyset$ is contained in $\widetilde{Q}$, and

$$
|\bar{Q}| \geq \frac{1}{4^{n}}\left|Q^{*}\right| \geq \frac{1}{22^{n}}|\widetilde{Q}| \geq \frac{1}{66^{n}}|\widetilde{\widetilde{Q}}| .
$$

Therefore,

$$
\left|m_{f}(\bar{Q})-c\right| \leq\left((f-c) \chi_{\bar{Q}}\right)^{*}(|\bar{Q}| / 2) \leq\left((f-c) \chi_{\widetilde{Q}}\right)^{*}\left(|\widetilde{\widetilde{Q}}| / 2 \cdot 66^{n}\right),
$$


and thus,

$$
\begin{aligned}
\inf _{c}\left((g-c) \chi_{Q^{\prime}}\right)^{*}\left(\left|Q^{\prime}\right| / 2\right) & =\inf _{c}\left((A f-c) \chi_{Q^{\prime}}\right)^{*}\left(\left|Q^{\prime}\right| / 2\right) \\
& \leq \inf _{c}\left((f-c) \chi_{\widetilde{Q}}\right)^{*}\left(|\widetilde{\widetilde{Q}}| / 2 \cdot 66^{n}\right) \\
& \leq \sup _{x \in F} M_{1 / 2 \cdot 66^{n} ; Q_{0}}^{\#} f(x) .
\end{aligned}
$$

Case 3. Let $Q^{\prime} \cap \Omega \neq \emptyset$, and suppose that for any $y \in Q^{\prime} \cap \Omega$ and any cube $Q^{*} \in \mathcal{Q}$ containing $y$ we have $\operatorname{diam}\left(Q^{\prime}\right)>\operatorname{diam}\left(Q^{*}\right) / 2$. Let $E$ be the union of such cubes $Q^{*}$, and let $\widetilde{Q}$ be a cube of minimal measure containing $E$ and $Q^{\prime}$. Then $\operatorname{dist}(\widetilde{Q}, F)<\operatorname{diam}(\widetilde{Q})$, and

$$
\operatorname{diam}\left(Q^{\prime}\right) \leq \operatorname{diam}(\widetilde{Q}) \leq 5 \operatorname{diam}\left(Q^{\prime}\right) .
$$

As in the previous case, there is a cube $\widetilde{\widetilde{Q}} \subset Q_{0}$ containing $\widetilde{Q}$ and such that $\widetilde{\widetilde{Q}} \cap F \neq \emptyset$ and $|\widetilde{\widetilde{Q}}| \leq 3^{n}|\widetilde{Q}|$.

Take an arbitrary constant $c$. To estimate $\left((g-c) \chi_{Q^{\prime}}\right)^{*}\left(\left|Q^{\prime}\right| / 2\right)$, we use the definition of the rearrangement. Let $E^{\prime}$ be any subset of $Q^{\prime}$ with $\left|E^{\prime}\right|=\left|Q^{\prime}\right| / 2$. Then either $\left|E^{\prime} \cap F\right| \geq\left|Q^{\prime}\right| / 4$ or $\left|E^{\prime} \cap \Omega\right| \geq\left|Q^{\prime}\right| / 4$. If $\left|E^{\prime} \cap F\right| \geq\left|Q^{\prime}\right| / 4$, then

$$
\begin{aligned}
\inf _{x \in E^{\prime}}|g-c| \leq \inf _{x \in E^{\prime} \cap F}|f-c| & \leq\left((f-c) \chi_{Q^{\prime}}\right)^{*}\left(\left|Q^{\prime}\right| / 4\right) \\
& \leq\left((f-c) \chi_{\widetilde{Q}}\right)^{*}\left(|\widetilde{\widetilde{Q}}| / 4 \cdot 15^{n}\right) .
\end{aligned}
$$

Assume that $\left|E^{\prime} \cap \Omega\right| \geq\left|Q^{\prime}\right| / 4$. Then, using (2.7), we get

$$
\begin{aligned}
\inf _{x \in E^{\prime}}|g-c| & \leq \inf _{x \in E^{\prime} \cap \Omega}|A f-c| \leq \inf _{x \in E^{\prime} \cap \Omega} m_{1 / 2}\left((f-c) \chi_{\widetilde{\widetilde{Q}}}\right) \\
& \leq\left(m_{1 / 2}\left((f-c) \chi_{\widetilde{\widetilde{Q}}}\right)\right)^{*}\left(\left|Q^{\prime}\right| / 4\right) \leq\left((f-c) \chi_{\widetilde{\widetilde{Q}}}\right)^{*}\left(\left|Q^{\prime}\right| / 8 \cdot 3^{n}\right) \\
& \leq\left((f-c) \chi_{\widetilde{\widetilde{Q}}}\right)^{*}\left(|\widetilde{\widetilde{Q}}| / 8 \cdot 45^{n}\right) .
\end{aligned}
$$

Therefore,

$$
\left((g-c) \chi_{Q^{\prime}}\right)^{*}\left(\left|Q^{\prime}\right| / 2\right) \leq\left((f-c) \chi_{\widetilde{Q}}\right)^{*}\left(|\widetilde{\widetilde{Q}}| / 8 \cdot 45^{n}\right),
$$

and thus,

$$
\inf _{c}\left((g-c) \chi_{Q^{\prime}}\right)^{*}\left(\left|Q^{\prime}\right| / 2\right) \leq \sup _{x \in F} M_{1 / 8 \cdot 45^{n} ; Q_{0}}^{\#} f(x) .
$$

Unifying all cases, we obtain

$$
\left\|M_{1 / 2 ; Q_{0}}^{\#} g\right\|_{\infty} \leq \sup _{x \in F} M_{1 / 8 \cdot 66^{n} ; Q_{0}}^{\#} f(x) .
$$

The lemma is proved.

The last lemma allows us to relate $\widetilde{M}_{\lambda, \omega}^{\#} f$ with $M_{\lambda}^{\#} f$ and the maximal function $P_{\lambda} \omega$ (cf. Section 2.3).

Lemma 5.3. For any measurable $f$, and any weight $\omega$,

$$
\left(\widetilde{M}_{\lambda, \omega}^{\#} f\right)_{\omega}^{*}(t) \leq c_{n}\left(M_{\lambda_{n}}^{\#} f P_{\lambda / 2} \omega\right)_{\omega}^{*}\left(\lambda t / c_{n}^{\prime}\right) \quad\left(0<t<\omega\left(\mathbb{R}^{n}\right), 0<\lambda<1\right) .
$$


Proof. Let $Q$ be any cube with $\omega(Q)>0$. Set

$$
\Omega=\left\{x \in Q: M_{\lambda_{n} ; Q}^{\#} f(x)>\left(M_{\lambda_{n} ; Q}^{\#} f\right)_{\omega}^{*}(\lambda \omega(Q) / 4)\right\} \quad \text { and } \quad F=Q \backslash \Omega,
$$

where $\lambda_{n}$ is the constant from Lemma 5.2. Then $\omega(F) \geq(1-\lambda / 4) \omega(Q)>0$, so that $|F|>0$. Let $g$ be the function from Lemma 5.2. By (2.1),

$$
\begin{aligned}
\inf _{c}\left((f-c) \chi_{Q}\right)_{\omega}^{*}(\lambda \omega(Q)) & \leq \inf _{c}\left((g-c) \chi_{Q}\right)_{\omega}^{*}(\lambda \omega(Q) / 2) \\
& +\left((f-g) \chi_{Q}\right)_{\omega}^{*}(\lambda \omega(Q) / 2) .
\end{aligned}
$$

The second term here is equal to zero, since $\omega(\operatorname{supp}(f-g)) \leq \omega(\Omega)<\omega(Q) / 2$. Let $E_{\lambda} \subset Q$ be any subset of minimal Lebesgue measure such that $\omega\left(E_{\lambda}\right)=\lambda \omega(Q) / 2$. Applying the John-Strömberg theorem (cf. (2.6)) and Lemma 5.2, we obtain

$$
\begin{aligned}
\inf _{c}\left((g-c) \chi_{Q}\right)_{\omega}^{*}(\lambda \omega(Q) / 2) & \leq\left(\left(g-m_{g}(Q)\right) \chi_{Q}\right)^{*}\left(\left|E_{\lambda}\right|\right) \\
& \leq c_{n}\left\|M_{1 / 2 ; Q}^{\#} g\right\|_{\infty} \log \frac{2|Q|}{\left|E_{\lambda}\right|} \\
& \leq c_{n} \sup _{x \in F} M_{\lambda_{n} ; Q}^{\#} f(x) \inf _{x \in Q} P_{\lambda / 2} \omega(x) \\
& \leq c_{n}\left(M_{\lambda_{n} ; Q}^{\#} f\right)_{\omega}^{*}(\lambda \omega(Q) / 4) \inf _{x \in Q} P_{\lambda / 2} \omega(x) .
\end{aligned}
$$

Therefore,

$$
\begin{aligned}
\inf _{c}\left((f-c) \chi_{Q}\right)_{\omega}^{*}(\lambda \omega(Q)) & \leq c_{n}\left(M_{\lambda_{n} ; Q}^{\#} f\right)_{\omega}^{*}(\lambda \omega(Q) / 4) \inf _{x \in Q} P_{\lambda / 2} \omega(x) \\
& \leq c_{n}\left(\left(M_{\lambda_{n}}^{\#} f P_{\lambda / 2} \omega\right) \chi_{Q}\right)^{*}(\lambda \omega(Q) / 4)
\end{aligned}
$$

and so,

$$
\widetilde{M}_{\lambda, \omega}^{\#} f(x) \leq c_{n} \widetilde{m}_{\lambda / 4, \omega}\left(M_{\lambda_{n}}^{\#} f P_{\lambda / 2} \omega\right)(x) .
$$

From this and from (5.5) we get (5.11).

Summarizing Corollary 5.1 and the last lemma, we have:

Theorem 5.4. If either $\omega\left(\mathbb{R}^{n}\right)<\infty$ or $\omega\left(\mathbb{R}^{n}\right)=\infty$ and $f_{\omega}^{*}(\infty)=0$, then

$$
\left(f-m_{f, \omega}\right)_{\omega}^{*}(t)^{\delta} \leq c_{n} \int_{c_{n}^{\prime} t}^{\omega\left(\mathbb{R}^{n}\right)}\left(M_{\lambda_{n}}^{\#} f P_{\lambda_{n}^{\prime}} \omega\right)_{\omega}^{*}(s)^{\delta} \frac{d s}{s} \quad\left(0<t<\omega\left(\mathbb{R}^{n}\right)\right),
$$

where $0<\delta \leq 1$, and

$$
\left\|f-m_{f, \omega}\right\|_{L_{\omega}^{p}} \leq c_{p, n}\left\|M_{\lambda_{n}}^{\#} f P_{\lambda_{n}^{\prime}} \omega\right\|_{L_{\omega}^{p}} \quad(0<p<\infty) .
$$

This theorem and (1.4) yield the following new weak-type and strong-type estimates for singular integrals.

Corollary 5.5. If either $\omega\left(\mathbb{R}^{n}\right)<\infty$ or $\omega\left(\mathbb{R}^{n}\right)=\infty$ and $(T f)_{\omega}^{*}(\infty)=0$, then

$$
\left(T f-m_{T f, \omega}\right)_{\omega}^{*}(t)^{\delta} \leq c_{n} \int_{c_{n}^{\prime} t}^{\omega\left(\mathbb{R}^{n}\right)}\left(M f P_{\lambda_{n}} \omega\right)_{\omega}^{*}(s)^{\delta} \frac{d s}{s} \quad\left(0<t<\omega\left(\mathbb{R}^{n}\right)\right),
$$

where $0<\delta \leq 1$, and

$$
\left\|T f-m_{T f, \omega}\right\|_{L_{\omega}^{p}} \leq c_{p, n}\left\|M f P_{\lambda_{n}} \omega\right\|_{L_{\omega}^{p}} \quad(0<p<\infty) .
$$


Using essentially the same methods one can deduce a little bit different proposition than Corollary [5.5 More precisely, given $0<\lambda<1$ and $r \geq 1$, define the operator $P_{\lambda}^{(r)} \omega$ by

$$
P_{\lambda}^{(r)} \omega(x)=\sup _{Q: x \in r Q} \log \left(1+|Q| /\left|E_{\lambda}\right|\right),
$$

where the supremum is taken over all cubes $Q$ such that $r Q$ contain $x$, and $E_{\lambda} \subset Q$ is any set of minimal Lebesgue measure such that $\omega\left(E_{\lambda}\right)=\lambda \omega(Q)$. Clearly, $P_{\lambda}^{(1)} \omega \equiv$ $P_{\lambda} \omega$, and $P_{\lambda}^{(r)} \omega \geq P_{\lambda} \omega$ for $r>1$.

Proposition 5.6. If either $\omega\left(\mathbb{R}^{n}\right)<\infty$ or $\omega\left(\mathbb{R}^{n}\right)=\infty$ and $(T f)_{\omega}^{*}(\infty)=0$, then for $r>1$ we have

$$
\left(T f-m_{T f, \omega}\right)_{\omega}^{*}(t)^{\delta} \leq c_{n, r} \int_{c_{n}^{\prime} t}^{\omega\left(\mathbb{R}^{n}\right)}\left(M\left(f P_{\lambda_{n}}^{(r)} \omega\right)\right)_{\omega}^{*}(s)^{\delta} \frac{d s}{s} \quad\left(0<t<\omega\left(\mathbb{R}^{n}\right)\right),
$$

where $0<\delta \leq 1$, and

$$
\left\|T f-m_{T f, \omega}\right\|_{L_{\omega}^{p}} \leq c_{p, n, r}\left\|M\left(f P_{\lambda_{n}}^{(r)} \omega\right)\right\|_{L_{\omega}^{p}} \quad(0<p<\infty) .
$$

Proof. Since the proof follows essentially the same ideas as before, we outline it briefly. First of all, standard arguments show that for any $x \in Q$,

$$
\begin{aligned}
\inf _{c}\left((T f-c) \chi_{Q}\right)_{\omega}^{*}(\lambda \omega(Q)) & \leq c_{r, n} M f(x) \\
& +\inf _{c}\left(\left(T\left(f \chi_{r Q}\right)-c\right) \chi_{Q}\right)_{\omega}^{*}(\lambda \omega(Q)) .
\end{aligned}
$$

Further, it is easy to see that instead of $(5.12)$ we can write

$$
\inf _{c}\left((f-c) \chi_{Q}\right)_{\omega}^{*}(\lambda \omega(Q)) \leq c_{n}\left(M_{\lambda_{n} ; Q}^{\#} f\right)_{\omega}^{*}(\lambda \omega(Q) / 4) \inf _{x \in r Q} P_{\lambda / 2}^{(r)} \omega(x) .
$$

From this we have

$$
\begin{aligned}
\inf _{c}\left(\left(T\left(f \chi_{r Q}\right)-c\right) \chi_{Q}\right)_{\omega}^{*}(\lambda \omega(Q)) & \leq c_{n}\left(\left(M_{r Q} f\right) \chi_{Q}\right)_{\omega}^{*}(\lambda \omega(Q) / 4) \inf _{x \in r Q} P_{\lambda / 2}^{(r)} \omega(x) \\
& \leq c_{n}\left(\left(M_{r Q}\left(f P_{\lambda / 2}^{(r)} \omega\right)\right) \chi_{Q}\right)_{\omega}^{*}(\lambda \omega(Q) / 4) .
\end{aligned}
$$

Therefore, in view of (5.17),

$$
\widetilde{M}_{\lambda, \omega}^{\#}(T f)(x) \leq c_{r, n} M f(x)+c_{n} \widetilde{m}_{\lambda / 4, \omega}\left(M\left(f P_{\lambda / 2}^{(r)} \omega\right)\right)(x),
$$

which, by (5.5), implies

$$
\begin{aligned}
\left(\widetilde{M}_{\lambda, \omega}^{\#}(T f)\right)_{\omega}^{*}(t) & \leq c_{r, n}(M f)_{\omega}^{*}(t / 2)+c_{n}\left(M\left(f P_{\lambda / 2}^{(r)} \omega\right)\right)_{\omega}^{*}\left(\lambda t / c_{n}^{\prime}\right) \\
& \leq c_{r, n}\left(M\left(f P_{\lambda / 2}^{(r)} \omega\right)\right)_{\omega}^{*}\left(\lambda t / c_{n}^{\prime}\right) .
\end{aligned}
$$

Now applying Corollary 5.1 completes the proof.

To realize the difference between Corollary [5.5] and Proposition [5.6] we invoke the Fefferman-Stein inequalities (4.7) and (4.8). Obviously, (5.14) combined with (4.7) gives

$$
\left\|T f-m_{T f, \omega}\right\|_{L_{\omega}^{p}} \leq c_{p, n} \int_{\mathbb{R}^{n}}|f|^{p} M\left(\omega\left(P_{\lambda_{n}} \omega\right)^{p}\right) d x \quad(1<p<\infty),
$$

while (5.16) combined with (4.7) yields

$$
\left\|T f-m_{T f, \omega}\right\|_{L_{\omega}^{p}} \leq c_{p, n, r} \int_{\mathbb{R}^{n}}|f|^{p}\left(P_{\lambda_{n}}^{(r)} \omega\right)^{p} M \omega d x \quad(1<p<\infty) .
$$


Observe also that (5.15), unlike (5.13), combined with (4.8) allows us to get the following weak-type estimate:

$$
\left(T f-m_{T f, \omega}\right)_{\omega}^{*}(t) \leq \frac{c_{n, r}}{t} \int_{\mathbb{R}^{n}}|f| P_{\lambda_{n}}^{(r)} \omega M \omega d x \quad\left(0<t<\omega\left(\mathbb{R}^{n}\right)\right) .
$$

It is still unknown whether the full analogue of (4.8) with singular integrals $T f$ (even with the Hilbert transform) instead of $M f$ holds (see [Pe]). Moreover, we do not know whether one can replace $P_{\lambda_{n}}^{(r)} \omega$ by $P_{\lambda_{n}} \omega$ on the right-hand side of (5.18).

\section{ACKNOWLEDGMENT}

The author is very grateful to the referee for the careful reading of the paper and for many helpful comments and remarks.

\section{REFERENCES}

[ABKP] J. Alvarez, R.J. Bagby, D.S. Kurtz and C. Pérez, Weighted estimates for commutators of linear operators, Studia Math. 104 (1993), no. 2, 195-209. MR94k:47044

[AP] J. Alvarez and C. Pérez, Estimates with $A_{\infty}$ weights for various singular integral operators, Boll. Un. Mat. Ital. A (7) 8 (1994), no. 1, 123-133. MR95f:42027

[AKMP] I.U. Asekritova, N.Ya. Krugljak, L. Maligranda and L.-E. Persson, Distribution and rearrangement estimates of the maximal function and interpolation, Studia Math. 124 (1997), no. 2, 107-132. MF 98g:46032

[BK] R.J. Bagby and D.S. Kurtz, Covering lemmas and the sharp function, Proc. Amer. Math. Soc. 93 (1985), 291-296. MR 86f:42011

[BDS] C. Bennett, R. DeVore and R. Sharpley, Weak- $L^{\infty}$ and BMO, Ann. of Math. 113 (1981), 601-611. MR82h:46047

[BS] C. Bennett and R. Sharpley, Interpolation of operators, Academic Press, New York, 1988. MR $89 \mathrm{e}: 46001$

[CF1] R. R. Coifman and C. Fefferman, Weighted norm inequalities for maximal functions and singular integrals, Studia. Math. 15 (1974), 241-250. MR50:10670

[CF2] A. Cordoba and C. Fefferman, A weighted norm inequality for singular integrals, Studia Math. 57 (1976), 97-101. MR 54:8132

[CP1] D. Cruz-Uribe and C. Pérez, Two-weight extrapolation via the maximal operator, J. Funct. Anal. 174 (2000), 1-17. MR2001g:42040

[CP2] D. Cruz-Uribe and C. Pérez, Two-weight, weak-type norm inequalities for fractional integrals, Calderón-Zygmund operators and commutators, Indiana Univ. Math. J. 49 (2000), no. 2, 697-721. MR 2001i:42021

[CR] K.M. Chong and N.M. Rice, Equimeasurable rearrangements of functions, Queen's Papers in Pure and Appl. Math. 28, Queen's University, Kingston, Ont., 1971. MF $51: 8357$

[FS1] C. Fefferman and E.M. Stein, Some maximal inequalities, Amer. J. Math. 93 (1971), 107-115. MR 44:2026

[FS2] C. Fefferman and E.M. Stein, $H^{p}$ spaces of several variables, Acta Math. 129 (1972), 137-193. MR.56:6263

[Ja] S. Janson, Mean oscillation and commutators of singular integral operators, Ark. Mat. 16 (1978), no. 2, 263-270. Mr 80j:42034

[JT] B. Jawerth and A. Torchinsky, Local sharp maximal functions, J. Approx. Theory 43 (1985), 231-270. MR86k:42034

[Jo] F. John, Quasi-isometric mappings, Seminari 1962 - 1963 di Analisi, Algebra, Geometria e Topologia, Rome, 1965. MR 32:8315

[JN] F. John and L. Nirenberg, On functions of bounded mean oscillation, Comm. Pure Appl. Math. 14 (1961), 415-426. MR.24:A1348

[Ku] D.S. Kurtz, Operator estimates using the sharp function, Pacific J. Math. 139 (1989), no. 2, 267-277. MR90g:42036

[L1] A.K. Lerner, On weighted estimates of non-increasing rearrangements, East J. Approx. 4 (1998), 277-290. MR99k:42043 
[L2] A.K. Lerner, On the John-Strömberg characterization of BMO for nondoubling measures, Real. Anal. Exchange, 28 (2003), no. 2, 649-660. MR2010341

[L3] A.K. Lerner, Weighted norm inequalities for the local sharp maximal function, J. Fourier Anal. Appl. 10 (2004), no. 5, 465-474.

[MMNO] J. Mateu, P. Mattila, A. Nicolau and J. Orobitg, BMO for nondoubling measures, Duke Math. J. 102 (2000), no.3, 533-565. MR.2001e:26019

[Pe] C. Pérez, Weighted norm inequalities for singular integral operators, J. London Math. Soc. 49 (1994), 296-308. MR94m:42037

[St] E.M. Stein, Harmonic Analysis, Princeton Univ. Press, Princeton, 1993. MR95c:42002

[Str] J.-O. Strömberg, Bounded mean oscillation with Orlicz norms and duality of Hardy spaces, Indiana Univ. Math. J. 28 (1979), 511-544. MF81f:42021

[W1] J.M. Wilson, Weighted inequalities for the dyadic square function without dyadic $A_{\infty}$, Duke Math. J. 55 (1987), 19-49. MR88d:42034

[W2] J.M. Wilson, A sharp inequality for the square function, Duke Math. J. 55 (1987), 879-887. MR89a:42029

[W3] J.M. Wilson, $L^{p}$ weighted norm inequalities for the square function, $0<p<2$, Ill. J. Math. 33 (1989), no.3, 361-366. MR90g:42037

[W4] J.M. Wilson, Weighted norm inequalities for the continuous square functions, Trans. Amer. Math. Soc. 314(1989), 661-692. MR91e:42025

Department of Mathematics, Bar-Ilan University, 52900 Ramat Gan, Israel

E-mail address: aklerner@netvision.net.il 